Pacific Journal of Mathematic

LINEAR GCD FeUATIONS
DAND hacoson 


\section{LINEAR GCD EQUATIONS}

\section{DAVID JACOBSON}

Let $R$ be a $G C D$ domain. Let $A$ be an $m \times n$ matrix and $B$ an $m \times 1$ matrix with entries in $R$. Let $c \neq 0, d \in R$. We consider the linear $G C D$ equation $G C D(A X+B, c)=d$. Let $S$ denote its set of solutions. We prove necessary and sufficient conditions that $S$ be nonempty. An element $t$ in $R$ is called a solution modulus if $X+t R^{n} \subseteq S$ whenever $X \in S$. We show that if $c / d$ is a product of prime elements of $R$, then the ideal of solution moduli is a principal ideal of $R$ and its generator $t_{0}$ is determined. When $R / t_{0} R$ is a finite ring, we derive an explicit formula for the number of distinct solutions $\left(\bmod t_{0}\right)$ of $G C D(A X+B, c)=d$.

1. Introduction. Let $R$ be a $G C D$ domain. As usual GCD $\left(a_{1}, \cdots, a_{m}\right)$ will denote a greatest common divisor of the finite sequence of elements $a_{1}, \cdots, a_{m}$ of $R$.

Let $A$ be an $m \times n$ matrix with entries $a_{i j}$ in $R$ and let $B$ be an $m \times 1$ matrix with entries $b_{i}$ in $R$ for $i=1, \cdots, m ; j=1, \cdots, n$. Let $c \neq 0, d$ be elements of $R$. In this paper we consider the "linear $G C D$ equation"

$$
\begin{aligned}
& G C D\left(a_{11} x_{1}+\cdots+a_{1 n} x_{n}+b_{1}, \cdots,\right. \\
& \left.a_{m 1} x_{1}+\cdots+a_{m n} x_{n}+b_{m}, c\right)=d .
\end{aligned}
$$

Letting $X$ denote the column of unknows $x_{1}, \cdots, x_{n}$ in (1.1), we shall find it convenient to abbreviate the equation (1.1) in matrix notation by

$$
G C D(A X+B, c)=d .
$$

Of course we allow a slight ambiguity in viewing (1.1) as an equation, since the GCD is unique only up to a unit.

Let $R^{n}$ denote the set of $n \times 1$ matrices with entries in $R$. We let $S \equiv S(A, B, c, d)$ denote the set of all solutions of (1.1), that is

$$
S=\left\{X \in R^{n} \mid G C D(A X+B, c)=d\right\} .
$$

If $S$ is nonempty, we say that (1.1) or (1.2) is solvable. Note that $X$ satisfies $G C D(A X+B, d)=d$ if and only if $X$ is a solution of the linear congruence system $A X+B \equiv 0(\bmod d)$.

We show in Proposition 1 that if (1.1) is solvable, then $d \mid c, A X+$ $B \equiv 0(\bmod d)$ has a solution and $G C D(A, d)=G C D(A, B, c)$. Here $G C D(A, d)=G C D\left(a_{11}, \cdots, a_{1 n}, \cdots, a_{m 1}, \cdots, a_{m n}, d\right)$ and $G C D(A, B, c)=$ $G C D\left(A, b_{1}, \cdots, b_{m}, c\right)$. Conversely we show in Proposition 3 that if 
the above conditions hold and $e=c / d$ is atomic, that is $e$ is a product of prime elements of $R$, then (1.1) is solvable. (Also see Proposition 4).

Let the solution set $S$ of (1.1) be nonempty. We say that $t$ in $R$ is a solution modulus of (1.1) if given $X$ in $S$ and $X \equiv X^{\prime}(\bmod t)$, then $X^{\prime}$ is in $S$. We let $M \equiv M(A, B, c, d)$ denote the set of all solution moduli of (1.1). We show in Theorem 2 that $M$ is an ideal of $R$ and if $e=c / d$ is atomic, then $M$ is actually a principal ideal generated by $d / g\left(p_{1} \cdots p_{k}\right)$, where $g=G C D(A, d)$ and $\left\{p_{1}, \cdots, p_{k}\right\}$ is a maximal set of nonassociated prime divisors of $e$ such that for each $p_{i}$, the system $A X+B \equiv 0\left(\bmod d p_{i}\right)$ is solvable. This generator $d / g\left(p_{1} \cdots p_{k}\right)$ denoted by $t_{0}$ is called the minimum modulus of (1.1).

In $\S 4$ we assume that $R / t_{0} R$ is a finite ring and we derive an explicit formula for the number of distinct equivalence classes of $R^{n}\left(\bmod t_{0}\right)$ comprising $S$. We denote this number by $N_{t_{0}} \equiv N_{t_{0}}(A, B, c, d)$. Let $A^{\prime}=A / g$ and $d^{\prime}=d / g$. Let $L=\left\{X+d^{\prime} R^{n} \mid A^{\prime} X \equiv 0\left(\bmod d^{\prime}\right)\right\}$ and $L_{i}=\left\{X+d^{\prime} R^{n} \mid A^{\prime} X \equiv 0\left(\bmod d^{\prime} p_{i}\right)\right\}$ for $i=1, \cdots, k$. In Theorem 3 we show that

$$
N_{t_{0}}=|L| \prod_{i=1}^{k}\left(\left|R / p_{i} R\right|^{n}-\left|R / p_{i} R\right|^{n-\left(r_{i}+s_{i}\right)}\right)
$$

where $r_{i}$ is rank $A^{\prime}\left(\bmod p_{i}\right)$ and $s_{i}$ is the dimension of the $R / p_{i} R$ vector space $L / L_{i}$.

The formula (1.3) is applied in some important cases. For example in Corollary 6 we determine $N_{t_{0}}$ when $R$ is a principal ideal domain.

This paper is an extension and generalization to GCD domains, of the results obtained over the ring of integers $Z$ in [2].

\section{Solvability of $G C D(A X+B, c)=d$.}

Proposition 1. If $G C D(A X+B, c)=d$ is solvable, then the following conditions hold.

(2.1) (i ) $d \mid c$,

(ii) $A X+B \equiv 0(\bmod d)$ is solvable,

(iii) $G C D(A, d)=G C D(A, B, c)$.

Proof. Let $X$ satisfy $G C D(A X+B, c)=d$. Then clearly (i) $d \mid c$ and (ii) $A X+B \equiv 0(\bmod d)$. Let $A X+B=d U$ where $U$ is an $m \times 1$ matrix with entries $u_{i}$ for $i=1, \cdots, m$. Then $G C D(d U, c)=$ $G C D\left(d u_{1}, \cdots, d u_{m}, c\right)=d$. Let $g=G C D(A, d)$ and $h=G C D(A, B, c)$. Then $B \equiv 0(\bmod g)$ as $A X-d U=B$ and $g \mid c$ as $d \mid c$, which shows that $g \mid h$. Also $d U \equiv 0(\bmod h)$, so that $h \mid G C D(d U, c)$, that is $h \mid d$. Thus $h \mid g$, which proves (iii).

Proposition 2. Let $e$ in $R$ have the following property 
( I ) $G C D(A X+B, e)=1$ is solvable whenever $G C D(A, B, e)=1$. Suppose that $c=d e, A X+B \equiv 0(\bmod d)$ is solvable and $G C D(A, d)=$ $G C D(A, B, c)$. Then $G C D(A X+B, c)=d$ is solvable.

Proof. There exist $X^{\prime}$ in $R^{n}$ and $V$ in $R^{m}$ such that $A X^{\prime}+B=d V$. Let $g=G C D(A, d)$ and let $A^{\prime}$ denote the matrix with entries $a_{i j} / g$ and $B^{\prime}$ the matrix with entries $b_{\imath} / g$ for $i=1, \cdots, m ; j=1, \cdots, n$. Then $A^{\prime} X^{\prime}+B^{\prime}=d^{\prime} V$ where $d^{\prime}=d / g$. We claim that $G C D\left(A^{\prime}, V, e\right)=1$. For let $h$ be any divisor of $G C D\left(A^{\prime}, V, e\right)$. Then $B^{\prime} \equiv 0(\bmod h)$ and $h \mid G C D\left(A^{\prime}, B^{\prime}, c^{\prime}\right)$. where $c^{\prime}=d^{\prime} e$. However, $G C D\left(A^{\prime}, B^{\prime}, c^{\prime}\right)=1$ as $g=G C D(A, B, c)$. Hence $h$ is a unit, that is $G C D\left(A^{\prime}, V, e\right)=1$. So by property (I), there is a $Y$ in $R^{n}$ such that $G C D\left(A^{\prime} Y+V, e\right)=1$. Thus $G C D\left(A\left(d^{\prime} Y\right)+d V, d e\right)=d$ and if we set $X=X^{\prime}+d^{\prime} Y$, then $G C D(A X+B, c)=d$, establishing the proposition.

We show in Proposition 3 that if $e$ is atomic, then $e$ satisfies property (I).

We require the following useful lemmas.

Lemma 1. Let $e=p_{1} \cdots p_{k}$ be a product of nonassociated prime elements $p_{1}, \cdots, p_{k}$ in $R$. If $G C D(A, B, e)=1$, then $G C D(A X+$ $B, e)=1$ is solvable.

Proof. Let $G C D(A, B, e)=1$. We use induction on $k$. Let $k=1$. If $G C D\left(B, p_{1}\right)=1$, then $X=0$ satisfies $G C D\left(A X+B, p_{1}\right)=1$. Suppose that $B \equiv 0\left(\bmod p_{1}\right)$. Then $G C D\left(A, p_{1}\right)=1$. Hence there is a $j$ such that $G C D\left(a_{1 j}, \cdots, a_{m j}, p_{1}\right)=1$. Let $X^{j}$ in $R^{n}$ have a 1 in the $j$ th position and o's elsewhere. Then $G C D\left(A X^{j}+B, p_{1}\right)=$ $G C D\left(A X^{j}, p_{1}\right)=1$. Thus $G C D\left(A X+B, p_{1}\right)=1$ is solvable. Now let $k>1$ and let $e^{\prime}=p_{1} \cdots p_{k-1}$. By the induction assumption there is $X^{\prime}$ in $R^{n}$ such that $G C D\left(A X^{\prime}+B, e^{\prime}\right)=1$. Let $B^{\prime}=A X^{\prime}+B$. We claim that $G C D\left(A e^{\prime}, B^{\prime}, p_{k}\right)=1$. If $G C D\left(A, p_{k}\right)=1$, then $G C D\left(A e^{\prime}\right.$, $\left.B^{\prime}, p_{k}\right)=1$. Suppose that $A \equiv 0\left(\bmod p_{k}\right)$. If $B^{\prime} \equiv 0\left(\bmod p_{k}\right)$, then $B \equiv 0\left(\bmod p_{k}\right)$, contradicting the hypothesis that $G C D(A, B, e)=1$. Hence $G C D\left(B^{\prime}, p_{k}\right)=1$, establishing the claim. So there exists a $Y$ in $R^{n}$ such that $G C D\left(\left(A e^{\prime}\right) Y+B^{\prime}, p_{k}\right)=1$. Let $X=X^{\prime}+e^{\prime} Y$. Then $X \equiv X^{\prime}\left(\bmod e^{\prime}\right)$ yields that $A X+B \equiv B^{\prime}\left(\bmod e^{\prime}\right)$. Thus $G C D(A X+$ $\left.B, e^{\prime}\right)=1$ since $G C D\left(B^{\prime}, e^{\prime}\right)=1$. Also

$$
G C D\left(A X+B, p_{k}\right)=G C D\left(\left(A e^{\prime}\right) Y+B^{\prime}, p_{k}\right)=1,
$$

:so that $G C D\left(A X+B, e^{\prime} p_{k}\right)=1$, completing the proof.

Lemma 2. Suppose that $e$ is an atomic element of $R$. 
Let $\left\{p_{1}, \cdots, p_{k}\right\}$ be a maximal set of nonassociated (*) prime divisors of e such that for each $p_{i}$, the system $A X+B \equiv 0\left(\bmod d p_{i}\right)$ is solvable.

Then $X$ is a solution of $G C D(A X+B, c)=d$ if and only if $G C D(A X+$ $\left.B, d e_{0}\right)=d$, where $c=$ de and $e_{0}=p_{1} \cdots p_{k}$.

Proof. Since $e$ is atomic, it is clear that we may select a set $\left\{p_{1}, \cdots, p_{k}\right\}$ as defined in $\left(^{*}\right)$. If this set is empty, we let $e_{0}=1$. Suppose that $X$ satisfies $G C D(A X+B, c)=d$. Then there is $U$ in $R^{m}$ such that $A X+B=d U$ and $G C D(U, e)=1$. Since $e_{0} \mid e$, $G C D\left(U, e_{0}\right)=1$ and thus $G C D\left(d U, d e_{0}\right)=d$, that is, $G C D(A X+$ $\left.B, d e_{0}\right)=d$.

Conversely let $X$ satisfy $G C D\left(A X+B, d e_{0}\right)=d$. Then $A X+$ $B=d U$ and $G C D\left(U, e_{0}\right)=1$. Suppose there is a prime $p \mid e$ and $U \equiv 0(\bmod p)$. Then $A X+B \equiv 0(\bmod d p)$ and the maximal property of the set $\left\{p_{1}, \cdots, p_{k}\right\}$ shows that $p$ is an associate of some $p_{i}$. So $U \equiv 0\left(\bmod p_{\imath}\right)$, contradicting that $G C D\left(U, e_{0}\right)=1$. Hence $G C D(U, p)=1$ for all primes $p \mid e$ and thus $G C D(U, e)=1$, that is $G C D(A X+B, c)=d$.

Proposition 3. Suppose that $c=d e, A X+B \equiv 0(\bmod d)$ is solvable and $G C D(A, d)=G C D(A, B, c)$. If $e$ is atomic, then $G C D(A X+$ $B, c)=d$ is solvable.

Proof. Let $e$ be atomic. By Proposition 2 it suffices to show that $e$ satisfies property (I). Thus let $G C D\left(A_{0}, B_{0}, e\right)=1$ where $A_{0}$ is an $m \times n$ matrix and $B_{0}$ is an $m \times 1$ matrix. By Lemma 2, $G C D\left(A_{0} X+B_{0}, e\right)=1$ is solvable if and only if $G C D\left(A_{0} X+B_{0}, e_{0}\right)=1$ is solvable where $e_{0}=p_{1} \cdots p_{k}$ is a product of nonassociated prime divisors of $e$. However by Lemma $1, G C D\left(A_{0} X+B_{0}, e_{0}\right)=1$ is solvable since $G C D\left(A_{0}, B_{0}, e_{0}\right)=1$. Thus (I) holds and $G C D(A X+B, c)=d$ is solvable.

Theorem 1. Let $R$ be a GCD domain. Consider the following condition

(II) $G C D\left(a_{1} x+b_{1}, \cdots, a_{m} x+b_{m}, c\right)=1$ is solvable if

$$
G C D\left(a_{1}, \cdots, a_{m}, b_{1}, \cdots, b_{m}, c\right)=1 ;
$$

(i ) If $R$ satisfies (II), then $G C D(A X+B, c)=1$ is solvable whenever $G C D(A, B, c)=1$.

(ii) If $R$ is a Bezout domain such that $G C D(a x+b, c)=1$ is solvable whenever $G C D(a, b, c)=1$, then $R$ satisfies (II).

Proof. (i ) Let $R$ satisfy (II). Let $G C D(A, B, c)=1$ where $A$ 
is an $m \times n$ matrix. We prove that $G C D(A X+B, c)=1$ is solvable by induction of $n$. For $n=1$, solvability is granted by the supposition (II). Let $n>1$ and let $A^{\prime}$ denote the $m \times(n-1)$ matrix with entries $a_{i, j+1}$ for $i=1, \cdots, m ; j=1, \cdots, n-1$. If $c^{\prime}=G C D\left(a_{11}, \cdots\right.$, $\left.a_{1 m}, c\right)$, then $G C D\left(A^{\prime}, B, c^{\prime}\right)=1$. Hence by the induction assumption, there exist $x_{2}, \cdots, x_{n}$ in $R$ such that $G C D\left(a_{12} x_{2}+\cdots+a_{1 n} x_{n}+\right.$ $\left.b_{1}, \cdots, a_{m 1} x_{2}+\cdots+a_{m n} x_{n}+b_{m}, c^{\prime}\right)=1$. If $b_{i}^{\prime}=a_{i 2} x_{2}+\cdots+a_{i n} x_{n}+b_{i}$ for $i=1, \cdots, m$, then $G C D\left(a_{11}, \cdots, a_{m 1}, b_{1}^{\prime}, \cdots, b_{m}^{\prime}, c\right)=1$. Thus by (II), there exists $x_{1}$ in $R$ such that $G C D\left(a_{11} x_{1}+b_{1}^{\prime}, \cdots, a_{m 1} x_{1}+b_{m}^{\prime}, c\right)=1$. So if $X$ in $R^{n}$ has entries $x_{1}, x_{2}, \cdots, x_{n}$, then $G C D(A X+B, c)=1$, completing the proof of (i).

(ii) Let $R$ be a Bezout domain, that is a domain in which every finitely generated ideal is principal. Suppose that $R$ has the property that $G C D(a x+b, c)=1$ is solvable if $G C D(a, b, c)=1$. Let

$$
G C D\left(a_{1}, \cdots, a_{m}, b_{1}, \cdots, b_{m}, c\right)=1 \text {. }
$$

Let $A$ and $B$ denote the $m \times 1$ matrices with entries $a_{1}, \cdots, a_{m}$ and $b_{1}, \cdots, b_{m}$ respectively. Then by [3, Theorem 3.5], there exists an invertible $m \times m$ matrix $P$ such that $P A$ has entries $a, 0, \cdots, o$. Also it is clear that $G C D(P A, P B, c)=1$. Let $P B$ have entries $b, b_{2}^{\prime}, \cdots, b_{m}^{\prime}$. Thus by hypothesis, $G C D\left(a x+b, c^{\prime}\right)=1$ is solvable where $c^{\prime}=$ $G C D\left(b_{2}^{\prime}, \cdots, b_{m}^{\prime}, c\right)$. Hence $G C D(A x+B, c)=1$ is solvable, that is $R$ satisfies (II).

As an immediate consequence of the preceding propositions and Theorem 1, we state

Proposition 4. Let $R$ be a UFD or a Bezout domain such that $G C D(a x+b, c)=1$ is solvable if $G C D(a, b, c)=1$. Then $G C D(A X+$ $B, c)=d$ is solvable if and only if $d \mid c, A X+B \equiv 0(\bmod d)$ is solvable and $G C D(A, d)=G C D(A, B, c)$.

We remark that we do not know whether there exists a GCD domain in which (II) is not valid. Any Bezout domain satisfying (II) is an elementary divisor domain [3, Theorem 5.2].

We conclude this section with the following result.

Proposition 5. Let $R$ be a Bezout domain. Suppose that (0) $G C D(a x+b, c)=1$ is solvable whenever $G C D(a, b)=1$ and $a \mid c$. Then $G C D(a x+b, c)=1$ is solvable whenever $G C D(a, b, c)=1$.

Proof. Let $G C D(a, b, c)=1$. If $a^{\prime}=G C D(a, c)$, then $G C D\left(a^{\prime}, b\right)=1$ and $a^{\prime} \mid c$. By the assumption (0), there is $x^{\prime}$ in $R$ such that $G C D\left(a^{\prime} x^{\prime}+b, c\right)=1$. If $u=a^{\prime} x^{\prime}+b$, then $a^{\prime} \mid(u-b)$ and since $R$ is a Bezout domain, there is an $x$ in $R$ such that $a x+b \equiv u(\bmod c)$. 
Thus $G C D(a x+b, c)=1$ since $G C D(u, c)=1$.

Let $a \mid c$ and let $\nu: R / c R \rightarrow R / a R$ be the epimorphism given by $\nu(r+c R)=r+a R$ for all $r$ in $R$. Let $G\left(\right.$ resp. $\left.G^{\prime}\right)$ denote the group of units of $R / c R$ (resp. $R / a R$ ). If $\nu^{\prime}: G \rightarrow G^{\prime}$ is the induced homomorphism, then note that $(0)$ is equivalent to the condition that $\nu^{\prime}(G)=G^{\prime} . \quad($ See $[5]$.

3. The minimum modulus. Let the solution set $S$ of $G C D(A X+B, c)=d$ be nonempty. Then

$$
M=\left\{t \in R \mid X+t R^{n} \subseteq S \text { for all } X \in S\right\}
$$

is the set of solution moduli of $G C D(A X+B, c)=d$.

Note that $c \in M$ for if $X \in S$ and $X \equiv X^{\prime}(\bmod c)$, then $A X+B \equiv$ $A X^{\prime}+B(\bmod c)$, so that $d=G C D\left(A X^{\prime}+B, c\right)$.

It is obvious that $M=R$, that is $S=R^{n}$ if and only if $d=$ $G C D(A, d)=G C D(A, B, c)$ and $G C D(A / d(X)+B / d, c / d)=1$ for all $X$ in $R^{n}$.

Theorem 2. Let $R$ be a GCD domain. Let $G C D(A X+B, c)=d$ be solvable. Let $e=c / d=\prod_{i=1}^{k} e_{i}$. Let $\hat{e}_{\imath}=e_{1} \cdots e_{i-1} e_{i+1} \cdots e_{k}$ for $i=1, \cdots, k$.

(1) $M$ is an ideal of $R$,

(2) $M \supseteqq \bigcap_{i=1}^{k} M_{i}$ where $M_{i}$ is the ideal of solution moduli for $G C D\left(A X+B, d e_{2}\right)=d$.

(3) If each $\hat{e}_{i}$ satisfies property (I) of Proposition 2, then $M=\bigcap_{i=1}^{k} M_{i}$ and $M$ is a principal ideal if each $M_{\imath}$ is principal.

(4) If $e$ is atomic, then $M$ is a principal ideal generated by $d / g\left(p_{1} \cdots p_{k}\right)$ where $g=G C D(A, d)$ and $\left\{p_{i}, \cdots, p_{k}\right\}$ is defined in $\left(^{*}\right)$ of Lemma 2.

Proof.

(1) As $S$ is nonempty, the set $M$ is well-defined and o, $c$ belong to $M$. Let $t_{1}, t_{2}$ be in $M$ and let $r \in R$. Let $X \in S$ and let $Y \in R^{n}$. Then $X+t_{1} Y \in S$ and hence $\left(X+t_{1} Y\right)+t_{2}(-Y) \in S$, that is $X+\left(t_{1}-t_{2}\right) Y \in S$ which shows that $t_{1}-t_{2} \in M$. Also $X+t_{1}(r Y) \in S$, that is $X+$ $\left(t_{1} r\right) Y \in S$. So $t_{1} r \in M$ and thus $M$ is an ideal of $R$.

(2) As $d \mid c$ we let $c=d e$. Let $S_{i}$ denote the solution set of $G C D\left(A X+B, d e_{i}\right)=d$ where $e=\prod_{i=1}^{k} e_{i}$. Then clearly $S=\bigcap_{i=1}^{k} S_{i}$. Let $t \in \bigcap_{i=1}^{c} M_{i}$. Let $X \in S$ and let $Y \in R^{n}$. Then $X+t Y \in \bigcap_{i=1}^{k} S_{i}$ since $X \in \bigcap_{i=1}^{k} S_{i}$. So $X+t Y \in S$, that is $t \in M$, which proves that $M \supseteqq \bigcap_{i=1}^{k} M_{i}$.

(3) Assume that each $\hat{e}_{i}$ satisfies property (I). We prove that $M \cong M_{i}$ for $i=1, \cdots, k$. As $g=G C D(A, d)=G C D(A, B, c)$, let $A^{\prime}=A / g, B^{\prime}=B / g$, and $d^{\prime}=d / g$. Let $i$ be fixed and let $X_{i} \in S_{i}$. 
Then $A^{\prime} X_{i}+B^{\prime}=d^{\prime} U$ where $G C D\left(U, e_{i}\right)=1$. We claim that $G C D\left(e_{i} A^{\prime}, U, \hat{e}_{i}\right)=1$. For let $h$ be a divisor of $G C D\left(e_{i} A^{\prime}, U, \hat{e}_{i}\right)$. Then $A^{\prime} \equiv 0(\bmod h)$ since $G C D\left(h, e_{i}\right)=1$. Thus $h \mid G C D\left(A^{\prime}, B^{\prime}, d^{\prime} e\right)$, that is $h \mid 1$. So by assumption there exists $X^{\prime}$ in $R^{n}$ such that

$$
G C D\left(\left(e_{i} A^{\prime}\right) X^{\prime}+U, \hat{e}_{i}\right)=1 .
$$

Let $X=X_{i}+d^{\prime} e_{i} X^{\prime}$. Then for $j=1, \cdots, k$,

$$
\begin{aligned}
& G C D\left(A^{\prime} X+B^{\prime}, d^{\prime} e_{j}\right) \\
& \quad=d^{\prime} G C D\left(\left(e_{i} A^{\prime}\right) X^{\prime}+U, e_{j}\right)=d^{\prime} .
\end{aligned}
$$

Hence $X \in \bigcap_{j=1}^{k} S_{j}$, that is $X \in S$. Now let $t \in M$ and let $Y \in R^{n}$. Then $X+t Y \in S$ and so $X+t Y \in S_{i}$. However, $X+t Y \equiv X_{i}+t Y\left(\bmod d^{\prime} e_{i}\right)$ and thus $X_{i}+t Y \in S_{i}$, that is $t \in M_{i}$, which proves that $M \subseteq M_{i}$. So by (2), $M=\bigcap_{i=1}^{k} M_{i}$. Moreover, if each $M_{i}$ is a principal ideal, say $M_{i}=t_{i} R$, then $\bigcap_{i=1}^{k} M_{i}$ is a principal ideal generated by the $\operatorname{LCM}\left(t_{1}, \cdots, t_{k}\right)$.

(4) Let $t$ be any element of $M$. We show that $d / g \mid t$ where $g=G C D(A, d)$. First note that $S$ is the solution set of $G C D\left(A^{\prime} X+\right.$ $\left.B^{\prime}, d^{\prime} e\right)=d^{\prime}$ where $A^{\prime}=A / g, B^{\prime}=B / g$, and $d^{\prime}=d / g$. Let $X \in S$ and let $A^{\prime} X+B^{\prime}=d^{\prime} U$. Then $G C D\left(A^{\prime}(X+t Y)+B^{\prime}, d^{\prime} e\right)=d^{\prime}$ for all $Y$ in $R^{n}$. So $G C D\left(\left(A^{\prime} t\right) Y+d^{\prime} U, d^{\prime} e\right)=d^{\prime}$ and thus $\left(A^{\prime} t\right) Y \equiv 0\left(\bmod d^{\prime}\right)$ for all $Y$ in $R^{n}$. Hence $A^{\prime} t \equiv 0\left(\bmod d^{\prime}\right)$ and since $G C D\left(A^{\prime}, d^{\prime}\right)=1$, it follows that $d^{\prime} \mid t$.

Now suppose that $e$ is atomic. By Lemma 2, $S$ is also the solution set of $G C D\left(A^{\prime} X+B^{\prime}, d^{\prime} e_{0}\right)=d^{\prime}$ where $e_{0}=p_{1} \cdots p_{k}$ and $\left\{p_{1}, \cdots, p_{k}\right\}$ is defined in $\left(^{*}\right)$. Thus $M$ is also the ideal of solution moduli of $G C D\left(A^{\prime} X+B^{\prime}, d^{\prime} e_{0}\right)=d^{\prime}$. Let $M_{i}^{\prime}$ denote the ideal of solution moduli of $G C D\left(A^{\prime} X+B^{\prime}, d^{\prime} p_{i}\right)=d^{\prime}$ for $i=1, \cdots, k$. Then Lemma 1 shows that (3) can be applied to yield that $M=\bigcap_{i=1}^{k} M_{i}^{\prime}$. We prove that each $M_{i}^{\prime}$ is a principal ideal generated by $d^{\prime} p_{i}$. Clearly $d^{\prime} p_{i} \in M_{i}^{\prime}$ for $i=1, \cdots, k$. Let $i$ be fixed and let $t$ be any element in $M_{i}^{\prime}$. Then as shown earlier, $d^{\prime} \mid t$ say $t=d^{\prime} t^{\prime}$. By $\left(^{*}\right)$ there exists $X$ in $R^{n}$ such that $A^{\prime} X+B^{\prime} \equiv 0\left(\bmod d^{\prime} p_{i}\right)$. Thus $G C D\left(A^{\prime}, p_{i}\right)=1$ since $G C D\left(A^{\prime}, B^{\prime}, d^{\prime} e\right)=1$. So there is a $j$ for which $G C D\left(A^{\prime} E_{j}, p_{i}\right)=1$ where $E_{j}$ is the $n \times 1$ matrix with 1 in the $j$ th position and o's elsewhere.

Now assume that $G C D\left(t^{\prime}, p_{i}\right)=1$. Let $X^{\prime}=X+t E_{j}$. Then $G C D\left(A^{\prime}\left(X^{\prime}-X\right), d^{\prime} p_{i}\right)=d^{\prime} G C D\left(t^{\prime} A^{\prime} E_{j}, p_{i}\right)=d^{\prime}$ since $G C D\left(t^{\prime} A^{\prime} E_{j}, p_{i}\right)=1$. So $G C D\left(A^{\prime} X^{\prime}-A^{\prime} X, d^{\prime} p_{i}\right)=d^{\prime}$ and thus $G C D\left(A^{\prime} X^{\prime}+B^{\prime}, d^{\prime} p_{i}\right)=d^{\prime}$ as $B \equiv-A^{\prime} X\left(\bmod d^{\prime} p_{i}\right)$. Hence $G C D\left(A^{\prime}\left(X^{\prime}+t\left(-E_{j}\right)\right)+B^{\prime}, d^{\prime} p_{i}\right)=d^{\prime}$ since $t \in M_{i}^{\prime}$. That is $G C D\left(A^{\prime} X+B^{\prime}, d^{\prime} p_{i}\right)=d^{\prime}$ and thus $d^{\prime} p_{i} \mid d^{\prime}$, which contradicts that $p_{i}$ is a nonunit. So the assumption that $G C D\left(t^{\prime}, p_{i}\right)=1$ is untenable, that is $p_{i} \mid t^{\prime}$. Thus $d^{\prime} p_{i} \mid t$ proving that 
$M_{i}^{\prime}=d^{\prime} p_{i} R$. However $M=\bigcap_{i=1}^{k} M_{i}^{\prime}$, so that $M$ is a principal ideal generated by the $L C M\left(d^{\prime} p_{1}, \cdots, d^{\prime} p_{k}\right)$, that is $M$ is generated by $d^{\prime} p_{1} \cdots p_{k}$.

The generator $d^{\prime} p_{1} \cdots p_{k}$ of $M$ is called the minimum modulus of $G C D(A X+B, d e)=d$.

4. The number of solutions with respect to a modulus. Let $G C D(A X+B, c)=d$ be solvable where $e=c / d$ is atomic. If $t$ in $R$ is a solution modulus of $G C D(A X+B, c)=d$, then $S$ consists of equivalence classes of $R^{n}(\bmod t)$. If $R / t R$ is also a finite ring, we let $N_{t} \equiv N_{t}(A, B, c, d)$ denote the number of distinct equivalence classes of $R^{n}(\bmod t)$ comprising $S$.

For $R / t R$ finite, let $|t|=|R / t R|$ denote the number of elements in $R / t R$. Note that if $t_{0} \mid t$, then each equivalence class of $R^{n}\left(\bmod t_{0}\right)$ consists of $\left|t / t_{0}\right|^{n}=\left(|t| /\left|t_{0}\right|\right)^{n}$ classes of $R^{n}(\bmod t)$. Thus if $t$ is a solution modulus and $t_{0}$ denotes the mininum modulus of $G C D(A X+$ $B, c)=d$, then $N_{t}=\left|t / t_{0}\right|^{n} N_{t_{0}}$. In Theorem 3, we explicitly determine $N_{t_{0}}$.

The following lemma is also of independent interest.

Lemma 3. Let $R$ be a GCD domain and suppose that $R / d R$ is a finite ring. Let $p_{1}, \cdots, p_{k}$ be nonassociated elements such that $R / p_{i} R$ is a finite field for $i=1, \cdots, k$. Let $A$ be an $m \times n$ matrix and let $r_{i}$ denote the rank of $A\left(\bmod p_{i}\right)$ for $i=1, \cdots, k$. Let $\mathscr{L}=\{X \in$ $\left.R^{n} \mid A X \equiv 0(\bmod d)\right\}$ and $L=\left\{X+d R^{n} \mid X \in \mathscr{L}\right\} . \quad$ Let $e_{0}=\prod_{i=1}^{k} p_{i}$ and let $\mathscr{L}^{\prime}=\left\{X \in R^{n} \mid A X \equiv 0\left(\bmod d e_{0}\right)\right\}$ and $L^{\prime}=\left\{X+d e_{0} R^{n} \mid X \in \mathscr{L}^{\prime}\right\}$. Let $\mathscr{L}_{i}=\left\{X \in R^{n} \mid A X \equiv 0\left(\bmod d p_{i}\right)\right\}$ and $L_{i}=\left\{X+d R^{n} \mid X \in \mathscr{L}_{i}\right\}$ for $i=1, \cdots, k$. Let $H=\left\{X+e_{0} R^{n} \mid X \in \mathscr{L}^{\prime}\right\}$ and $H_{i}=\left\{X+p_{i} R^{n} \mid X \in \mathscr{L}_{i}\right\}$ for $i=1, \cdots, k$. Then

$$
\left|L^{\prime}\right|=|L||H|
$$

and

$$
|H|=\prod_{i=1}^{k}\left|H_{i}\right|
$$

$L / L_{i}$ is an $R / p_{i} R$ vector space of dimension $s_{i}$ and $\left|H_{i}\right|=\left|R / p_{i} R\right|^{n-\left(r_{i}+s_{i}\right)}$ for $i=1, \cdots, k$.

$s_{i}=0$ if and only if for each $X$ in $\mathscr{L}$ there exists $X^{\prime}$ in $\mathscr{L}_{i}$ such that $X^{\prime} \equiv X(\bmod d)$.

If $G C D\left(d, p_{i}\right)=1$, then $s_{i}=0$.

$|L|=1$ if and only if $n=\operatorname{rank} A(\bmod p)$ for each prime $p \mid d$. 


\section{Proof.}

(1) In the obvious way, $L, L^{\prime}$, and $H$ are $R$-modules. Let $\sigma: L^{\prime} \rightarrow H$ denote the $R$-homomorphism defined by $\sigma\left(X+d e_{0} R^{n}\right)=$ $X+e_{0} R^{n}$ for all $X$ in $\mathscr{L}^{\prime}$. Then clearly $\operatorname{Ker} \sigma=\left\{e_{0} Y+d e_{0} R^{n} \mid Y \in \mathscr{L}\right\}$ so that $L \cong \operatorname{Ker} \sigma$ under the $R$-isomorphism $\tau: L \rightarrow \operatorname{Ker} \sigma$ defined by $\tau\left(Y+d R^{n}\right)=e_{0} Y+d e_{0} R^{n}$ for all $Y$ in $\mathscr{L}$. Thus $\left|L^{\prime}\right|=|L||H|$ since $\operatorname{Im} \sigma=H$. We now show that $H$ is isomorphic to $\bigoplus_{i=1}^{k} H_{i}$, the direct sum of the $R$-modules $H_{i}$. Let $\gamma: H \rightarrow \bigoplus_{i=1}^{k} H_{i}$ denote the $R$-homomorphism defined by $\gamma\left(X+e_{0} R^{n}\right)=\left(X+p_{1} R^{n}, \cdots, X+p_{k} R^{n}\right)$ for all $X$ in $\mathscr{L}^{\prime}$. If $X+e_{0} R^{n} \in \operatorname{Ker} \gamma$, then $X \equiv 0\left(\bmod p_{i}\right)$ for $i=1, \cdots, k$, that is $X \equiv 0\left(\bmod e_{0}\right)$, which shows that $\gamma$ is $1-1$. To show that $\operatorname{Im} \gamma=\bigoplus_{i=1}^{k} H_{i}$, let $X_{i} \in \mathscr{L}_{i}$ for $i=1, \cdots, k$. Since $R / d R$ is finite, it is easy to verify that $d$ is atomic. Thus let $d=d_{0} \prod_{i=1}^{k} p_{i}^{m_{i}}$ where $m_{i} \geqq 0$ and $G C D\left(d_{0}, p_{i}\right)=1$. By the Chinese remainder theorem there exists $X$ in $R^{n}$ such that $X \equiv 0\left(\bmod d_{0}\right)$ and $X \equiv X_{i}\left(\bmod p_{i}^{m_{i}+1}\right)$ for $i=1, \cdots, k$. However, $A X_{i} \equiv 0\left(\bmod p_{i}^{m_{i}+1}\right)$ for $i=1, \cdots, k$, so that $A X \equiv 0 \bmod \left(d_{0} \prod_{i=1}^{k} p_{i}^{m_{i}+1}\right)$, that is $A X \equiv 0\left(\bmod d e_{0}\right)$. Thus $X+$ $e_{0} R^{n} \in H$ and $\gamma\left(X+e_{0} R^{n}\right)=\left(X_{1}+p_{1} R^{n}, \cdots, X_{k}+p_{k} R^{n}\right)$. Hence $\gamma$ is an isomorphism and $|H|=\prod_{i=1}^{k}\left|H_{2}\right|$.

(2) Let $L_{i}^{\prime}=\left\{X+d p_{i} R^{n} \mid X \in \mathscr{L}_{\imath}\right\}$ for $i=1, \cdots, k$. Let $i$ be fixed. Let $\nu: L_{i}^{\prime} \rightarrow L_{i}$ denote the $R$-homomorphism defined by $\nu\left(X+d p_{i} R^{n}\right)=X+d R^{n}$ for all $X$ in $\mathscr{L}_{i}$. Then clearly Ker $\nu=$ $\left\{d Y+d p_{i} R^{n} \mid A Y \equiv 0\left(\bmod p_{i}\right)\right\}$ and it follows that

$$
|\operatorname{Ker} \nu|=\left|R / p_{i} R\right|^{n-r_{i}} \equiv\left|p_{i}\right|^{n-r_{i}}
$$

where $r_{i}=\operatorname{rank} A\left(\bmod p_{i}\right)$. Thus $\left|L_{i}^{\prime}\right|=\left|p_{i}\right|^{n-r_{i}}\left|L_{i}\right|$ since $\operatorname{Im} \nu=L_{i}$. However by (1), $\left|L_{i}^{\prime}\right|=|L|\left|H_{i}\right|$. Also since $L_{i}$ is an $R$-submodule of $L$, the quotient module $L / L_{i}$ is defined and $|L|=\left|L_{i}\right|\left|L / L_{i}\right|$. Thus we obtain that $\left|H_{i}\right|\left|L / L_{i}\right|=\left|p_{i}\right|^{n-r_{i}}$. We now show that $L / L_{i}$ is an $R / p_{i} R$ vector space. Let $\langle X\rangle=X+d R^{n}$ for $X$ in $R^{n}$. Then $L / L_{i}=\left\{\langle X\rangle+L_{i} \mid X \in \mathscr{L}\right\}$. For $r$ in $R$, let $\bar{r}=r+p_{i} R$ in $R / p_{i} R$. We define $\bar{r}\left(\langle X\rangle+L_{i}\right)=\langle r X\rangle+L_{i}$ for all $r$ in $R$ and $X$ in $\mathscr{L}$. We claim that this is a well-defined $R / p_{i} R$ multiplication on $L / L_{i}$. For let $\bar{r}=\bar{r}^{\prime}$ and $\langle X\rangle+L_{i}=\left\langle X^{\prime}\right\rangle+L_{i}$, where $r, r^{\prime} \in R$ and $X, X^{\prime} \in \mathscr{L}$. Then $r-r^{\prime} \equiv \operatorname{oo}\left(\bmod p_{i}\right)$ and $\langle X\rangle-\left\langle X^{\prime}\right\rangle \in L_{i}$, that is $\left\langle X-X^{\prime}\right\rangle \in L_{i}$. Thus there exists $Y$ in $\mathscr{L}_{i}$ such that $\left\langle X-X^{\prime}\right\rangle=$ $\langle Y\rangle$. We must show that $\langle r X\rangle+L_{i}=\left\langle r^{\prime} X^{\prime}\right\rangle+L_{i}$, that is $\left\langle r X-r^{\prime} X^{\prime}\right\rangle \in L_{i}$. We write $r X-r^{\prime} X^{\prime}=\left(r-r^{\prime}\right) X+r^{\prime}\left(X-X^{\prime}\right)$. However, $X-X^{\prime} \equiv Y(\bmod d)$ and thus $r\left(X-X^{\prime}\right) \equiv r Y(\bmod d)$. So $r X-r^{\prime} X^{\prime} \equiv\left(r-r^{\prime}\right) X+r Y(\bmod d)$ and $\left(r-r^{\prime}\right) X+r Y \in \mathscr{L}_{i}$. Hence $\left\langle r X-r^{\prime} X^{\prime}\right\rangle \in L_{i}$, which establishes the claim. It follows immediately that $L / L_{i}$ is an $R / p_{i} R$ vector space since $L / L_{i}$ is an $R$-module.

Let $s_{i}$ denote the dimension of the $R / p_{i} R$ vector space $L / L_{i}$. 
Then $\left|L / L_{i}\right|=\left|p_{i}\right|^{s_{i}}$ and as $\left|H_{i}\right|\left|L / L_{i}\right|=\left|p_{i}\right|^{n-r_{i}}$, we obtain that $\left|H_{i}\right|\left|p_{i}\right|^{s_{i}}=\left|p_{i}\right|^{n-r_{i}}$. Thus $o \leqq s_{i} \leqq n-r_{i}$ and $\left|H_{i}\right|=\left|p_{i}\right|^{n-\left(r_{i}+s_{i}\right)}$, which completes the proof of (2).

(3) As $|L|=\left|L_{i}\right|\left|p_{i}\right|^{s_{i}}$, it is immediate that $s_{i}=0$ if and only if $L=L_{i}$, that is if and only if for each $X$ in $\mathscr{L}$ there exists $X^{\prime}$ in $\mathscr{L}_{i}$ such that $X^{\prime} \equiv X(\bmod d)$.

(4) Suppose that $G C D\left(d, p_{i}\right)=1$. Let $X \in \mathscr{L}$. By the Chinese remainder theorem there exists $X^{\prime}$ in $R^{n}$ such that $X^{\prime} \equiv X(\bmod d)$ and $X^{\prime} \equiv 0\left(\bmod p_{i}\right)$. Thus $A X^{\prime} \equiv 0\left(\bmod d p_{i}\right)$, so that $s_{i}=0$ by (3).

(5) Let $p$ be a prime dividing $d$ and let $d=d_{1} p$. Then $L=$ $\left\{X+d_{1} p R^{n} \mid X \in \mathscr{L}\right\}$. However as shown in the proof of (2), $|L|=$ $|p|^{n-r_{0}}\left|L_{0}\right|$ where $r_{0}=\operatorname{rank} A(\bmod p)$ and $L_{0}=\left\{X+d_{1} R^{n} \mid X \in \mathscr{L}\right\}$. Thus if $|L|=1$, then $n=\operatorname{rank} A(\bmod p)$ for any prime $p \mid d$. The converse is trivial.

TheOREM 3. Let $R$ be a GCD domain. Let $G C D(A X+B, c)=d$ be solvable and suppose that $e=c / d$ is atomic. Let $A^{\prime}=A / g$ and $d^{\prime}=d / g$ where $g=G C D(A, d)$. Let $t_{0}=d^{\prime} \prod_{i=1}^{k} p_{i}$ denote the minimum modulus of $G C D(A X+B, c)=d$ where $\left\{p_{1}, \cdots, p_{k}\right\}$ is defined in (*) of Lemma 2. Suppose that $R / t_{0} R$ is a finite ring. Let $L=$ $\left\{X+d^{\prime} R^{n} \mid A^{\prime} X \equiv 0\left(\bmod d^{\prime}\right)\right\}$ and $L_{i}=\left\{X+d^{\prime} R^{n} \mid A^{\prime} X \equiv 0\left(\bmod d^{\prime} p_{i}\right)\right\}$ for $i=1, \cdots, k$. Then

$$
N_{t_{0}}=|L| \prod_{i=1}^{k}\left(\left|p_{i}\right|^{n}-\left|p_{i}\right|^{n-\left\langle r_{i}+s_{i}\right)}\right)
$$

where $r_{i}$ denotes $\mathrm{rank} A^{\prime}\left(\bmod p_{i}\right)$ and $s_{i}$ denotes the dimension of the $R / p_{i} R$ vector space $L / L_{i}$.

Proof. Let $S$ denote the solution set of $G C D(A X+B, c)=d$. As $g=G C D(A, B, c)$, let $B^{\prime}=B / g$. Then by Lemma $2, S$ is also the solution set of $G C D\left(A^{\prime} X+B, d^{\prime} e_{0}\right)=d^{\prime}$ where $e_{0}=\prod_{i=1}^{k} p_{\imath}$. Let $\mathscr{S}$ denote the set of $X$ in $R^{n}$ such that $A^{\prime} X+B^{\prime} \equiv 0\left(\bmod d^{\prime}\right)$. Let $\mathscr{S}_{i}$ denote the set of $X$ in $R^{n}$ such that $A^{\prime} X+B^{\prime} \equiv 0\left(\bmod d^{\prime} p_{i}\right)$ for $i=1, \cdots, k$. It is clear that $S=\mathscr{S} \backslash \bigcup_{i=1}^{k} \mathscr{S}_{2}$. Let $T_{0}=\{X+$ $\left.t_{0} R^{n} \mid X \in S\right\}$. Then $\left|T_{0}\right|$ is what we have denoted by $N_{t_{0}}$. Also let $T=\left\{X+t_{0} R^{n} \mid X \in \mathscr{S}\right\}$ and $T_{i}=\left\{X+t_{0} R^{n} \mid X \in \mathscr{S}_{i}\right\}$ for $i=1, \cdots, k$. Hence $T_{0}=T \backslash \bigcup_{i=1}^{k} T_{i}$ and by the method of inclusion and exclusion

$$
N_{t_{0}}=\left|T_{0}\right|=\sum_{I}(-1)^{\mid I_{1}}\left|T_{I}\right|
$$

where the summation is over all subsets $I$ of

$$
I_{k}=\{1, \cdots, k\} \text { and } T_{I}=\bigcap_{i=1} T_{i} .
$$

Now let $\mathscr{S}_{I}=\bigcap_{i \in I} \mathscr{S}_{i}$ and $d_{I}^{\prime}=d^{\prime} \prod_{i \in I} p_{i}$ for each subset $I$ of 
$I_{k}$. Then it is easy to see that $\mathscr{S}_{I}$ is the set of $X$ in $R^{n}$ such that $A^{\prime} X+B^{\prime} \equiv 0\left(\bmod d_{I}^{\prime}\right)$ and $T_{I}=\left\{X+t_{0} R^{n} \mid X \in \mathscr{S}_{I}\right\}$. Let $T_{I}^{\prime}=\{X+$ $\left.d_{I}^{\prime} R^{n} \mid X \in \mathscr{S}_{I}\right\}$ and let $I^{\prime}=I_{k} \backslash I$. Then $\left|T_{I}\right|=\left|T_{I}^{\prime}\right| \Pi_{i \in I^{\prime}}\left|p_{i}\right|^{n}$, since $X+d_{I}^{\prime} R^{n}$ consists of $\left|t_{0} / d_{I}^{\prime}\right|^{n}=\prod_{i \in I^{\prime}}\left|p_{i}\right|^{n}$ distinct classes of $R^{n}\left(\bmod t_{0}\right)$.

Let $\mathscr{L}_{I}$ denote the set of $X$ in $R^{n}$ such that $A^{\prime} X \equiv 0\left(\bmod d_{I}^{\prime}\right)$. Let $L_{I}^{\prime}=\left\{X+d_{I}^{\prime} R^{n} \mid X \in \mathscr{L}_{I}\right\} . \quad$ As $\mathscr{S}_{i}$ is nonempty for $i=1, \cdots, k$, an argument involving the Chinese remainder theorem shows that each $\mathscr{S}_{I}$ is nonempty. Hence it follows that $\left|T_{I}^{\prime}\right|=\left|L_{I}^{\prime}\right|$. Let $L=$ $\left\{X+d^{\prime} R^{n} \mid X \in \mathscr{L}_{\phi}\right\}$ and $L_{i}=\left\{X+d^{\prime} R^{n} \mid X \in \mathscr{L}_{|i|}\right\}$ for $i=1, \cdots, k$. Then (1) and (2) of Lemma 3 yield that $\left|L_{I}^{\prime}\right|=|L| \prod_{i \in I}\left|p_{i}\right|^{n-\left(r_{i}+s_{i}\right)}$ where $r_{i}=\operatorname{rank} A^{\prime}\left(\bmod p_{i}\right)$ and $s_{i}=$ dimension of the $R / p_{i} R$ vector space $L / L_{i}$.

Hence by (4.2),

$$
N_{t_{0}}=|L| \sum_{I}(-1)^{|I|} \prod_{i \in I}\left|p_{i}\right|^{n-\left(r_{i}+s_{i}\right)} \prod_{i \in I^{\prime}}\left|p_{i}\right|^{n}
$$

where the summation is over all subsets $I$ of $I_{k}$ and $I^{\prime}=I_{k} \backslash I$. Thus we may write

$$
N_{t_{0}}=|L| \prod_{i=1}^{k}\left|p_{i}\right|^{n} \sum_{I}(-1)^{|I|} \prod_{i \in I}\left|p_{i}\right|^{-\left(r_{i}+s_{i}\right)}
$$

where the summation is over all subsets $I$ of $I_{k}$. However,

$$
\prod_{i=1}^{k}\left(1-\left|p_{i}\right|^{-\left(r_{i}+s_{i}\right)}\right)=\sum_{I}(-1)^{|I|} \prod_{i \in I}\left|p_{i}\right|^{-\left(r_{i}+s_{i}\right)},
$$

which yields the formula (4.1) for $N_{t_{0}}$. This completes the proof of the theorem.

We remark that if $p_{i}^{m_{i}}$ is the highest power of $p_{i}$ dividing $d^{\prime}$, then $s_{i}$ is also the dimension of the $R / p_{i} R$ vector space $K_{i}^{0} / K_{i}$ where $K_{i}^{0}=\left\{X+p_{i}^{m_{i}} R^{n} \mid A^{\prime} X \equiv 0\left(\bmod p_{i}^{m_{i}}\right)\right\}$ and

$$
K_{i}=\left\{X+p_{i}^{m_{i}} R^{n} \mid A^{\prime} X \equiv 0\left(\bmod p_{i}^{m_{i}+1}\right)\right\} .
$$

Also note that $r_{i} \geqq 1$ for $i=1, \cdots, k$.

In Corollaries 1 and 2 , the notation is the same as in Theorem 3.

Corollary 1. Let $G C D(A X+B, c)=d$ be solvable and suppose that $e=c / d$ is atomic. Let $R / t_{0} R$ be finite where $t_{0}=d^{\prime} \prod_{i=1}^{k} p_{\imath}$ is the minimum modulus of $G C D(A X+B, c)=d$.

(i ) If $G C D\left(d^{\prime}, e\right)=1$, then

$$
N_{t_{0}}=|L| \prod_{i=1}^{k}\left(\left|p_{i}\right|^{n}-\left|p_{i}\right|^{n-r_{i}}\right)
$$

(ii) If $|L|=1$, then 


$$
N_{t_{0}}=\prod_{i=1}^{k}\left(\left|p_{i}\right|^{n}-\left|p_{i}\right|^{n-r_{i}}\right)
$$

where $r_{i}=n$ if $p_{i} \mid d^{\prime}$.

(iii) If $n^{\prime}=\operatorname{rank} A^{\prime}\left(\bmod p_{i}\right)$ for $i=1, \cdots, k$, where $n^{\prime}$ denotes the smaller of $m$ and $n$, then

$$
N_{t_{0}}=|L| \prod_{i=1}^{k}\left(\left|p_{i}\right|^{n}-\left|p_{i}\right|^{n-n^{\prime}}\right) .
$$

(iv) $N_{t_{0}}=1$ if and only if (a) $|L|=1$ and there exists no prime $p \mid e$ such that $A X+B \equiv 0(\bmod d p)$ is solvable, or (b) $n=1$ and $|p|=2$ for any prime $p \mid e$ such that $A X+B \equiv 0(\bmod d p)$ is solvable.

Proof.

(i) If $G C D\left(d^{\prime}, p_{i}\right)=1$, then (4) of Lemma 3 shows that $s_{i}=0$ in (4.1). Hence if $G C D\left(d^{\prime}, e\right)=1$, then $s_{i}=0$ for $i=1, \cdots, k$, which yields (4.3).

(ii) Suppose that $|L|=1$. If $p_{i} \mid d^{\prime}$, then $n=r_{2}$ by (5) of Lemma 3 and thus $s_{i}=0$ since $s_{i} \leqq n-r_{i}$. However if $G C D\left(d^{\prime}, p_{\imath}\right)=1$, then $s_{i}=0$, so that (4.4) is immediate from (4.1).

In particular if $d=1$, then $N_{t_{0}}$ is given by (4.4). If $A^{\prime}$ is invertible $\left(\bmod d^{\prime}\right)$, then (4.4) also applies.

(iii) If $n=r_{i}$, then $s_{i}=0$. If $m=r_{i}$, then the criterion in (3) shows that $s_{i}=0$. Thus (4.5) follows from (4.1).

(iv) Suppose that $N_{t_{0}}=1$. Then by (4.1), $|L|=1$ and thus $s_{i}=0$ for $i=1, \cdots, k$. If $p_{i}$ is a prime dividing $e$ such that $A X+B \equiv 0\left(\bmod d p_{i}\right)$ is solvable, then $\left|p_{i}\right|^{n}-\left|p_{i}\right|^{n-r_{i}}=1$, so that $n=r_{i}=1$ and $\left|p_{i}\right|=2$. Thus either (a) or (b) holds. Conversely if (a) holds, then $N_{t_{0}}=1$. If $n=1$, then clearly $|L|=1$ and hence (b) implies that $N_{t_{0}}=1$.

Corollary 2. Let $G C D(A X+B, c)=d$ be solvable and let $e=c / d$. Suppose that $R / c R$ is a finite ring. Then

$$
N_{c}=|L||g e|^{n} \prod_{i=1}^{k}\left(1-\left|p_{i}\right|^{-\left\langle r_{i}+s_{i}\right\rangle}\right) \text {. }
$$

Proof. Since $R / c R$ is finite, $e$ is atomic. Thus $t_{0}=d^{\prime} \prod_{i=1}^{k} p_{i}$ is the minimum modulus of $G C D(A X+B, c)=d$. Also $R / t_{0} R$ is finite since $t_{0} \mid c$, so that $N_{t_{0}}$ is given by (4.1). However $N_{c}=\left|c / t_{0}\right|^{n} N_{t_{0}}$, which yields the result (4.6).

Corollary 3. Suppose that $R / c R$ is a finite ring. Then $G C D\left(a_{1} x_{1}+\cdots+a_{n} x_{n}+b, c\right)=d$ is solvable if and only if $d \mid c$ and $G C D\left(a_{1}, \cdots, a_{n}, d\right)=G C D\left(a_{1}, \cdots, a_{x}, b, c\right)$. Let $a_{j}^{\prime}=a_{j} / g$ for $j=1, \cdots, n$ 
where $g=G C D\left(a_{1}, \cdots, a_{n}, d\right)$. Let $\left\{p_{1}, \cdots, p_{k}\right\}$ be a maximal set of nonassociated prime divisors of $e=c / d$ such that $G C D\left(a_{1}^{\prime}, \cdots, a_{n}^{\prime}, p_{i}\right)=1$ for $i=1, \cdots, k$. Then

$$
N_{c}=|c|^{n-1}|g e| \prod_{i=1}^{k}\left(1-\left|p_{i}\right|^{-1}\right) .
$$

Proof. Suppose that $c=d e$ and $g=G C D\left(a_{1}, \cdots, a_{n}, b, c\right)$. Since $R / c R$ is finite, $d$ is atomic and $R / p R$ is a finite field for any prime $p \mid d$. Hence as $g \mid b$, a standard argument shows that $a_{1} x_{1}+\cdots+$ $a_{n} x_{n}+b \equiv \mathrm{o}(\bmod d)$ is solvable and has $|g||d|^{n-1}$ distinct solutions $(\bmod d)$. Thus $G C D\left(a_{1} x_{1}+\cdots+a_{n} x_{n}+b, c\right)=d$ is solvable since $e$ is atomic. Let $d^{\prime}=d / g$ and $b^{\prime}=b / g$. Since $G C D\left(a_{1}^{\prime}, \cdots, a_{n}^{\prime}, d^{\prime} p_{i}\right)=1$ and $R / d^{\prime} p_{i} R$ is finite, $a_{1}^{\prime} x_{1}+\cdots+a_{n}^{\prime} x_{n}+b^{\prime} \equiv 0\left(\bmod d^{\prime} p_{i}\right)$ is solvable for $i=1, \cdots, k$. It follows that $t_{0}=d^{\prime} \prod_{i=1}^{k} p_{i}$ is the minimum modulus of $G C D\left(a_{1} x_{1}+\cdots+a_{n} x_{n}+b, c\right)=d$. Let $A^{\prime}$ denote the $1 \times n$ matrix $\left(a_{1}^{\prime}, \cdots, a_{n}^{\prime}\right)$. Then rank $A^{\prime}\left(\bmod p_{i}\right)=1$ for $i=1, \cdots, k$. Also $a_{1}^{\prime} x_{1}+\cdots+a_{n}^{\prime} x_{n} \equiv \mathrm{o}\left(\bmod d^{\prime}\right)$ has $\left|d^{\prime}\right|^{n-1}$ distinct solutions $\left(\bmod d^{\prime}\right)$. Thus by (iii) of Corollary 1 ,

$$
N_{t_{0}}=\left|d^{\prime}\right|^{n-1} \prod_{i=1}^{k}\left(\left|p_{i}\right|^{n}-\left|p_{i}\right|^{n-1}\right),
$$

which yields (4.7).

Corollary 4. Suppose that $R / c R$ is a finite ring where $c=$ de. Let $g=G C D\left(a_{1}, \cdots, a_{m}, d\right)$ and $a_{i}^{\prime}=a_{i} / g$ for $i=1, \cdots, m$. Then $G C D\left(a_{1} x+b_{1}, \cdots, a_{m} x+b_{m}, c\right)=d$ is solvable if and only if

(1) $G C D\left(a_{i}, d\right) \mid b_{i}$ for $i=1, \cdots, m$,

(2) $a_{i}^{\prime} b_{j} \equiv a_{j}^{\prime} b_{i}(\bmod d)$ for $1 \leqq i<j \leqq m$,

(3) $g=G C D\left(a_{1}, \cdots, a_{m}, b_{1}, \cdots, b_{m}, c\right)$.

Let $\left\{p_{1}, \cdots, p_{k}\right\}$ be a maximal set of nonassociated prime divisors of $e$ such that for each $p_{h}, G C D\left(a_{i}, d p_{h}\right) \mid b_{i}$ for $i=1, \cdots, m$ and $a_{i}^{\prime} \equiv a_{j}^{\prime} b_{i}\left(\bmod d p_{h}\right)$ for $1 \leqq i<j \leqq m$. Then

$$
N_{c}=|g e| \prod_{h=1}^{k}\left(1-\left|p_{h}\right|^{-1}\right) \text {. }
$$

Proof. Let $A$ and $B$ denote the $m \times 1$ matrices with entries $a_{1}, \cdots, a_{m}$ and $b_{1}, \cdots, b_{m}$ respectively. Since $R / d R$ is finite, the reader may easily verify that the system $A x+B \equiv 0(\bmod d)$ is solvable if and only if (1) and (2) hold. Thus as $e$ is atomic, $G C D(A x+B, c)=d$ is solvable if and only if (1), (2), and (3) hold. Let $G C D(A x+B, c)=d$ be solvable and let $d^{\prime}=d / g$. Then it follows that $t_{0}=d^{\prime} \prod_{h=1}^{k} p_{h}$ is the minimum modulus of $G C D(A x+B, c)=d$. Let $A^{\prime}$ denote the $m \times 1$ matrix with entries $a_{1}^{\prime}, \cdots, a_{m}^{\prime}$. Then $\operatorname{rank} A^{\prime}\left(\bmod p_{i}\right)=1$ for 
$i=1, \cdots, k$. Also the system $A^{\prime} x \equiv 0\left(\bmod d^{\prime}\right)$ has only the solution $x \equiv \mathrm{o}\left(\bmod d^{\prime}\right)$. Thus by (iii) of Corollary $1, N_{t_{0}}=\prod_{h=1}^{k}\left(\left|p_{h}\right|-1\right)$. Hence $N_{c}=|g e| \prod_{h=1}^{k}\left(1-\left|p_{h}\right|^{-1}\right)$.

COROLlary 5. Let $c=$ de where $e$ is atomic. Let $g=G C D\left(a_{1}\right.$, $\left.\cdots, a_{n}, d\right)$ and $d^{\prime}=d / g$. Suppose that $R / d^{\prime} R$ is a finite ring. Then $G C D\left(a_{1} x_{1}+b_{1}, \cdots, a_{n} x_{n}+b_{n}, c\right)=d$ is solvable if and only if $G C D\left(a_{j}, d\right) \mid b_{j}$ for $j=1, \cdots, n$ and $g=G C D\left(a_{1}, \cdots, a_{n}, b_{1}, \cdots, b_{n}, c\right)$. Suppose that $R /\left(\prod_{i=1}^{k} p_{i}\right) R$ is finite where $\left\{p_{1}, \cdots, p_{k}\right\}$ is a maximal set of nonassociated prime divisors of $e$ such that for each $p_{i}$, $G C D\left(a_{j}, d p_{i}\right) \mid b_{j}$ for $j=1, \cdots, n$. Then $t_{0}=d^{\prime} \prod_{i=1}^{k} p_{i}$ is the minimum modulus of $G C D\left(a_{1} x_{1}+b_{1}, \cdots, a_{n} x_{n}+b_{n}, c\right)=d$. Let $d_{j}=G C D\left(a_{j}, d\right)$ and $d_{j}^{\prime}=d_{j} / g$ for $j=1, \cdots, n$. Then

$$
N_{t_{0}}=\prod_{j=1}^{n}\left|d_{j}^{\prime}\right| \prod_{i=1}^{k}\left(\left|p_{i}\right|^{n}-\left|p_{i}\right|^{n-t_{i}}\right)
$$

where $t_{\imath}$ denotes the number of $j$ in $\{1, \cdots, n\}$ for which

$$
G C D\left(\frac{a_{j}}{d_{j}}, p_{i}\right)=1 \text {. }
$$

Proof. Suppose that $d_{j} \mid b_{j}$ for $j=1, \cdots, n$. Let $a_{j}^{\prime}=a_{j} / g$ and $b_{j}^{\prime}=b_{j} / g$ for $j=1, \cdots, n$. Let $A$ and $A^{\prime}$ denote the $n \times n$ diagonal matrices with entries $a_{1}, \cdots, a_{n}$ and $a_{1}^{\prime}, \cdots, a_{n}^{\prime}$ respectively. Let $B$ and $B^{\prime}$ denote the $n \times 1$ matrices with entries $b_{1}, \cdots, b_{n}$ and $b_{1}^{\prime}, \cdots, b_{n}^{\prime}$ respectively. Then the system $A^{\prime} X+B^{\prime} \equiv 0\left(\bmod d^{\prime}\right)$ is solvable since $G C D\left(a_{j}^{\prime}, d^{\prime}\right) \mid b_{j}^{\prime}$ for $j=1, \cdots, n$ and $R / d^{\prime} R$ is finite. Thus the system $A X+B \equiv 0(\bmod d)$ is solvable. Hence if $g=G C D\left(a_{1}, \cdots, a_{n}, b_{1}, \cdots, b_{n}, c\right)$, then $G C D(A X+B, c)=d$ is solvable.

Assume that $G C D(A X+B, c)=d$ is solvable. It follows that $t_{0}=d^{\prime} \prod_{i=1}^{k} p_{i}$ is the minimum modulus of $G C D(A X+B, c)=d$. Let $L=\left\{X+d^{\prime} R^{n} \mid A^{\prime} X \equiv 0\left(\bmod d^{\prime}\right)\right\}$. Let

$$
\mathscr{L}_{i}=\left\{X \in R^{n} \mid A^{\prime} X \equiv 0\left(\bmod d^{\prime} p_{i}\right)\right\}
$$

and $L_{\imath}=\left\{X+d^{\prime} R^{n} \mid X \in \mathscr{L}_{i}\right\}$ for $i=1, \cdots, k$. Then by (4.1),

$$
N_{t_{0}}=|L| \prod_{i=1}^{k}\left(\left|p_{i}\right|^{n}-\left|p_{i}\right|^{n-\left(r_{i}+s_{i}\right)}\right)
$$

where $r_{i}=\operatorname{rank} A^{\prime}\left(\bmod p_{i}\right)$ and $s_{i}$ is the dimension of the $R / p_{i} R$ vector space $L / L_{i}$. Clearly $|L|=\prod_{j=1}^{n}\left|d_{j}^{\prime}\right|$ since $d_{j}^{\prime}=G C D\left(\alpha_{j}^{\prime}, d^{\prime}\right)$ for $j=1, \cdots, n$. Let $L_{i}^{\prime}=\left\{X+d^{\prime} p_{i} R^{n} \mid X \in \mathscr{L}_{i}\right\}$ and $H_{i}=\{X+$ $\left.p_{i} R^{n} \mid X \in \mathscr{L}_{i}\right\}$ for $i=1, \cdots, k$. Then (1) and (2) of Lemma 3 show that $\left|L_{i}^{\prime}\right|=|L|\left|H_{i}\right|$ where $\left|H_{i}\right|=\left|p_{i}\right|^{n-\left(r_{i}+s_{i}\right)}$ for $i=1, \cdots, k$. However, $G C D\left(a_{j}^{\prime}, d^{\prime} p_{i}\right)=d_{j}^{\prime} G C D\left(a_{j} / d_{j}, p_{i}\right)$ and thus 


$$
\left|L_{i}^{\prime}\right|=|L| \prod_{j=1}^{n}\left|G C D\left(\frac{a_{j}}{d_{j}}, p_{i}\right)\right|
$$

for $i=1, \cdots, k$. Hence $\left|p_{i}\right|^{n-\left\langle r_{i}+s_{i}\right\rangle}=\prod_{j=1}^{n}\left|G C D\left(a_{j} / d_{j}, p_{i}\right)\right|$ and thus $\left|p_{i}\right|^{n-\left(r_{i}+s_{i}\right)}=\left|p_{i}\right|^{n-t_{i}}$, since $t_{i}$ is the number of $j$ in $\{1, \cdots, n\}$ for which $G C D\left(a_{j} / d_{j}, p_{i}\right)=1$. So $t_{i}=r_{i}+s_{i}$ for $i=1, \cdots, k$, which yields (4.8).

Note that if $R / c R$ is finite, then

$$
N_{c}=\prod_{j=1}^{n}\left|d_{j} e\right| \prod_{i=1}^{k}\left(1-\left|p_{i}\right|^{-t_{i}}\right) .
$$

Corollary 6. Let $R$ be a principal ideal domain. Let $A$ be an $m \times n$ matrix of rank $r$ and let $\alpha_{1}, \cdots, \alpha_{r}$ be the invariant factors of $A$. Let $B$ be an $m \times 1$ matrix and let $(A: B)$ have rank $r^{\prime}$ and invariant factors $\beta_{1}, \cdots, \beta_{r^{\prime}}$. Then $G C D(A X+B, c)=d$ is solvable if and only if (1) $d \mid c$, (2) $G C D\left(\alpha_{1}, d\right)=G C D\left(\beta_{1}, c\right)$, (3) $G C D\left(\alpha_{j}, d\right)=G C D\left(\beta_{j}, d\right)$ for $j=1, \cdots, r \quad$ and $\quad \beta_{r^{\prime}} \equiv \operatorname{ood}(\bmod d) \quad$ if $r^{\prime}=r+1$.

Let $\left\{p_{1}, \cdots, p_{k}\right\}$ be a maximal set of nonassociated prime divisors of $e=c / d$ such that each $p_{i}$ satisfies $\left(3^{\prime}\right) G C D\left(\alpha_{j}, d p_{i}\right)=G C D\left(\beta_{j}, d p_{i}\right)$ for $j=1, \cdots, r$ and $\beta_{r^{\prime}} \equiv \mathrm{o}\left(\bmod d p_{i}\right)$ if $r^{\prime}=r+1$. Let $d_{j}=G C D\left(\alpha_{j}, d\right)$ for $j=1, \cdots, r$ and $d^{\prime}=d / d_{1}$. Then $t_{0}=d^{\prime} \Pi_{i=1}^{k} p_{i}$ is the minimum modulus of $G C D(A X+B, c)=d$. Suppose that $R / t_{0} R$ is finite. Then

$$
N_{t_{0}}=\left|d^{\prime}\right|^{n-r} \prod_{j=1}^{r}\left|d_{j}^{\prime}\right| \prod_{i=1}^{k}\left(\left|p_{i}\right|^{n}-\left|p_{i}\right|^{n-t_{i}}\right)
$$

where $d_{j}^{\prime}=d_{j} / d_{1}$ and $t_{2}$ denotes the largest $j$ in $\{1, \cdots, r\}$ for which $G C D\left(\alpha_{j} / d_{j}, p_{i}\right)=1$.

Proof. Since $R$ is a principal ideal domain, it is well-known that there exist invertible matrices $P$ and $Q$ such that $P A Q=A_{0}$ where $A_{0}$ is an $m \times n$ matrix in "diagonal form", with nonzero entries $\alpha_{1}, \cdots, \alpha_{r}$ and $\alpha_{j} \mid \alpha_{j^{\prime}}$ if $j<j^{\prime}$. The elements $\alpha_{1}, \cdots, \alpha_{r}$ are called the invariant factors of $A$ and $\alpha_{j}=D_{j} / D_{j-1}$ where $D_{j}$ denotes the $G C D$ of the determinants of all the $j \times j$ submatrices of $A$. Clearly $G C D(A, d)=G C D\left(\alpha_{1}, \cdots, \alpha_{r}, d\right)$, that is $G C D(A, d)=G C D\left(\alpha_{1}, d\right)$ since $\alpha_{1} \mid \alpha_{j}$ for $j=1, \cdots, r$. Similarly $G C D(A, B, c)=G C D\left(\beta_{1}, c\right)$. However, it is also well-known that the system $A X+B \equiv 0(\bmod d)$ is solvable if and only if condition (3) holds (see [4]). Thus $G C D(A X+$ $B, c)=d$ is solvable if and only if (1), (2), and (3) hold.

Let $G C D(A X+B, c)=d$ be solvable and let $c=d e$. Then $t_{0}=d^{\prime} \prod_{i=1}^{k} p_{i}$ is the minimum modulus of $G C D(A X+B, c)=d$. Suppose that $R / t_{0} R$ is finite. Let $S$ denote the set of $X$ in $R^{n}$ such 
that $G C D(A X+B, c)=d$. Let $P B=B_{0}$ and let $S^{\prime}$ denote the set of $Y$ in $R^{n}$ such that $G C D\left(A_{0} Y+B_{0}, c\right)=d$. Then clearly $X \in S$ if and only if $Y=Q^{-1} X \in S^{\prime}$. Thus $G C D(A X+B, c)=d$ and $G C D\left(A_{0} Y+\right.$ $\left.B_{0}, c\right)=d$ have the same ideal of solution moduli. Let $T_{0}=\{X+$ $\left.t_{0} R^{n} \mid X \in S\right\}$ and $T_{0}^{\prime}=\left\{Y+t_{0} R^{n} \mid Y \in S^{\prime}\right\}$. Then the mapping $f: T_{0} \rightarrow T_{0}^{\prime}$ is a bijection, where $f\left(X+t_{0} R^{n}\right)=Q^{-1} X+t_{0} R^{n}$ for all $X$ in $S$. Hence $\left|T_{0}\right|=\left|T_{0}^{\prime}\right|$, that is $N_{t_{0}}=\left|T_{0}^{\prime}\right|$. Let $B_{0}$ have entries $b_{1}^{0}, \cdots, b_{m}^{0}$ and let $c_{0}=G C D\left(b_{r+1}^{0}, \cdots, b_{m}^{0}, c\right)$. Then $S^{\prime}$ is the set of solutions of the linear $G C D$ equation

$$
\begin{gathered}
G C D\left(\alpha_{1} y_{1}+b_{1}^{0}, \cdots, \alpha_{r} y_{r}+b_{r}^{0}, o \cdot y_{r+1}+o,\right. \\
\left.\cdots, o \cdot y_{n}+o, c_{0}\right)=d .
\end{gathered}
$$

Thus $t_{0}=d^{\prime} \prod_{i=1}^{k} p_{i}$ is also the minimum modulus of (4.10) and hence by (4.8) of Corollary 5 ,

$$
N_{t_{0}}=\left|d^{\prime}\right|^{n-r} \prod_{j=1}^{r}\left|d_{j}^{\prime}\right| \prod_{i=1}^{k}\left(\left|p_{i}\right|^{n}-\left|p_{i}\right|^{n-t_{i}}\right)
$$

where $d_{j}^{\prime}=d_{j} / d_{1}$ and $t_{i}$ is the largest $j$ in $\{1, \cdots, r\}$ for which $G C D\left(\alpha_{j} / d_{j}, p_{i}\right)=1$ since $\alpha_{j} / d_{j} \mid \alpha_{j^{\prime}} / d_{j^{\prime}}$ if $j<j^{\prime}$.

If $R / c R$ is finite, then

$$
N_{c}=|c|^{n-r} \prod_{j=1}^{r}\left|d_{j} e\right| \prod_{i=1}^{k}\left(1-\left|p_{i}\right|^{-t_{i}}\right) .
$$

Finally we remark that the formula for $N_{t_{0}}$ in (4.1) applies to the class $\mathscr{D}$ of $G C D$ domains $R$ which contain at least one element $p$ such that $R / p R$ is a finite field. Some immediate examples are the integers $\boldsymbol{Z}$, the localizations $\boldsymbol{Z}_{(p)}$ at primes $p$ in $\boldsymbol{Z}$ and $F[X]$ where $F$ is a finite field.

However, an example of such a ring $R$ in $\mathscr{D}$ which is not a $P I D$ is the subring $R$ of $\boldsymbol{Q}[X]$ consisting of all polynomials whose constant term is in $Z$. Indeed $R$ is a Bezout domain which cannot be expressed as an ascending union of PID's [1]. Clearly if $p$ is a prime in $Z$, then $R / p R$ is isomorphic to the finite field $\boldsymbol{Z} / p \boldsymbol{Z}$.

We are also indebted to Professor W. Heinzer for the following construction of a ring $R$ in $\mathscr{D}$ which is a $U F D$ but not a PID. Let $F$ be a finite field. Let $Y$ be an element of the formal power series ring $F[[X]]$ such that $X$ and $Y$ are algebraically independent over $F$. Let $V$ denote the rank one discrete valuation ring $F[[X]] \cap F(X, Y)$ and let $R=F[X, Y][1 / X] \cap V$. Then $R / X R$ is isomorphic to $F$ and $R$ is a $U F D$.

\section{REFERENCES}

1. P. M. Cohn, Bezout rings and their subrings, Proc. Camb. Phil. Soc., 64 (1968), 251-264. 
2. D. Jacobson and K. S. Williams, On the solution of linear GCD equations, Pacific J. Math., 39 (1971), 187-206.

3. I. Kaplansky, Elementary divisors and modules, Trans. Amer. Math. Soc., 66 (1949), 464-491.

4. H. J. S. Smith, On systems of linear indeterminate equations and congruences, Phil. Trans. London, 151 (1861), 293-326. (Collected Mathematical Papers Vol. 1, Chelsea, N.Y.), (1965), 367-409.

5. R. Spira, Elementary problem no. E1730, Amer. Math. Monthly, 72 (1965), 907.

Received February 22, 1974 and in revised form September 3, 1974. This research was supported in part by NRC Grant-A8749.

UNIVERSITY OF NEW BRUNSWICK 



\section{PACIFIC JOURNAL OF MATHEMATICS}

EDITORS

RICHARD ARENS (Managing Editor)

University of California

Los Angeles, Calıfornia 90024

R. A. Beaumont

University of Washington

Seattle, Washington 98105
J. DugundJI

Department of Mathematics

University of Southern California

Los Angeles, California 90007

D. Gilbarg and J. Milgram

Stanford University

Stanford, California 94305

\section{ASSOCIATE EDITORS}
E. F. BECKENBACH
B. H. NeumanN
F. WOLF
K. YOSHIDA

\section{SUPPORTING INSTITUTIONS}

UNIVERSITY OF BRITISH COLUMBIA

CALIFORNIA INSTITUTE OF TECHNOLOGY

UNIVERSITY OF CALIFORNIA

MONTANA STATE UNIVERSITY

UNIVERSITY OF NEVADA

NEW MEXICO STATE UNIVERSITY

OREGON STATE UNIVERSITY

UNIVERSITY OF OREGON

OSAKA UNIVERSITY
UNIVERSITY OF SOUTHERN CALIFORNIA

STANFORD UNIVERSITY

UNIVERSITY OF TOKYO

UNIVERSITY OF UTAH

WASHINGTON STATE UNIVERSITY

UNIVERSITY OF WASHINGTON

AMERICAN MATHEMATICAL SOCIETY NAVAL WEAPONS CENTER 


\section{Pacific Journal of Mathematics}

\section{Vol. 55, No. $1 \quad$ September, 1974}

Robert Lee Anderson, Continuous spectra of a singular symmetric

differential operator on a Hilbert space of vector-valued functions . . . $\quad 1$

Michael James Cambern, The isometries of $L^{p}(X, K) \ldots \ldots \ldots \ldots \ldots . . \ldots$

R. H. Cameron and David Arne Storvick, Two related integrals over spaces of continuous functions ................................

Gary Theodore Chartrand and Albert David Polimeni, Ramsey theory and

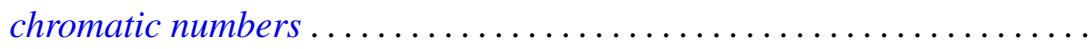

John Deryck De Pree and Harry Scott Klein, Characterization of collectively compact sets of linear operators ...................

John Deryck De Pree and Harry Scott Klein, Semi-groups and collectively compact sets of linear operators ....................... 55

George Epstein and Alfred Horn, Chain based lattices.............. 65

Paul Erdős and Ernst Gabor Straus, On the irrationality of certain series . . 85

Zdeněk Frolík, Measurable uniform spaces................... 93

Stephen Michael Gagola, Jr., Characters fully ramified over a normal

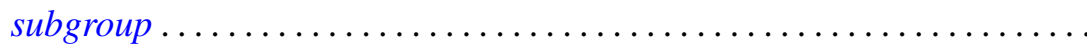

Frank Larkin Gilfeather, Operator valued roots of abelian analytic

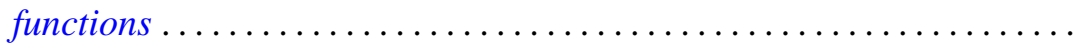

D. S. Goel, A. S. B. Holland, Cyril Nasim and B. N. Sahney, Best approximation by a saturation class of polynomial operators

James Secord Howland, Puiseux series for resonances at an embedded

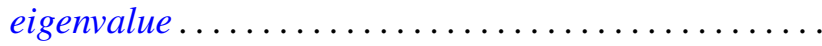

David Jacobson, Linear GCD equations .................

P. H. Karvellas, A note on compact semirings which are multiplicative

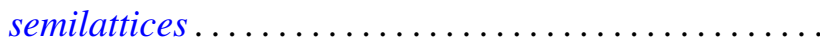

Allan Morton Krall, Stieltjes differential-boundary operators. II . .

D. G. Larman, On the inner aperture and intersections of convex sets

S. N. Mukhopadhyay, On the regularity of the $P^{n}$-integral and its application to summable trigonometric series ....... .

Dwight Webster Read, On $(J, M, m)$-extensions of Boolean algebras ....

David Francis Rearick, Multiplicativity-preserving arithmetic power series.

Indranand Sinha, Characteristic ideals in group algebras

Charles Thomas Tucker, II, Homomorphisms of Riesz spaces . . .

Kunio Yamagata, The exchange property and direct sums of indecomposable injective modules. 